tion does not ocour in dogs under similar circum. stances. Walther has shown that rabbits and dogs differ greatly in their reaction toward acids. While in rabbits, a sufficient quantity of acid proves fatal, dogs are almost immune against it. The difference in their reaction toward acids is caused by the greater amount of ammonia which is produced through the influence of the acids in dogs. It is not necessary to seek the cause for edema in the formation of acids directly, but it must be acknowledged that certain chemic peculiarities exist in these animals which determine the difference in the formation of edema of the lung.

Sigmund Mayer and von Sahli demonstrated that curare lessens the liability of the formation of edema of the lung, while muscular spasms favored its production. Their explanation has obstruction, increased blood pressure, and filtration for its basis; however, Professor Loeb gives a more interesting cause for this difference. The muscles are the principal seat of the oxidation processes and, therefore, in these structures the acids must be formed which are produced by a lack of oxygen. When curare is given, the metabolic processes of the muscles are reduced, and less poisonous matter circulates through the heart and lungs. Muscular spasms, on the other hand, cause an increase of these injurious substances and are, therefore, more liable to produce edema.

According to Professor Loeb, the cause of edema lies in the chemic changes which occur in the tissues, and which are mostly due to a lack of oxygen. These changes lead to an increase of osmotic pressure, which is greater in the tissues than in the blood and lymph. Morphologic alterations of the vessels are also a consequence of the chemic exciting cause. Such conditions as blood stasis, inflammation, etc., are only the primary causes which give rise to chemic changes and consequently edema.

For fifty years after Bright and Bartels, the filtration theory has held undisputed sway. Nobody seems to have considered any other force than mere filtration as an explanation for the minute phenomena of edema. Through the work of Cohnheim and his pupils, however, a future was given to the theory. Bright's theory was disproved and new factors were found which promised to be worthy of careful research. The frequent suggestion that an alteration of the vessel walls and blood pressure are the means by which the fluid escapes from the vessels, is only a part of the filtration theory. It is hardly possible that any other change than a mechanical one was thought of when an alteration of the vessel walls was mentioned. The work of Cohnheim, Welch and others has really been done in the interest of the filtration theory, the chemic side of the formation of edema having been neglected. Only recently the theory has found a physical and chemic basis through the work of Loeb. The theory of edema stands today where the theory of absorption stood before Haidenhain's classic paper was published. In spite of the many attacks upon Haidenhain's explanation, the merits of his work have not been lessened. In fact, the criticisms of the extreme physicists have only served to strengthen the belief that the cells play an important rôle and that osmotic pressure is not the only factor to be considered in absorption. Just as important is the physiologic state of the absorbing membrane. The same ond has been reached in the theory of secretion through the work of Ludwig.
Why should edema be such a passive process? Undoubtedly it is true that not so much stress can be placed upon cell activity in edema as in secretion and absorption, naturally edema would be a more passive phenomenon. Just as secretion and absorption depend upon the physiologic condition of the cell, so does edema depend upon the pathologic state of the cell. Still it is best not to conjecture, but to await further developments of the theory of edema.

\section{RETROMAXILLARY GROWTHS.}

OPERATIVE TREATMENT, ESPECIALLY OF NASOPHARYNGEAL FIBROMATA.

\section{BY CARL BECK, M.D.}

CHICAgo.

Among the tumors of the nasopharyngeal cavities which are amenable to operative treatment, the nasopharyngeal fibromata, or so-called nasopharyngeal polypi, are the most important. Sarcomata and carcinomata of this region may also be treated surgically, but when one considers how unfavorable the prog. nosis is, even in the case of a very good operative result, one must acknowledge that it is inexpedient to undertake so serious an operation for the sake of such a trifling benefit. Butler, who has paid particular attention to this question, finding an operative mortality of 30 per cent. and such a small number of cases free from recurrence after three years, arrived at the conclusion that the operation must be condemned unless there is a reasonable hope of obtaining better results in the future. In addition to the nasopharyngeal poly pi and malignant growths, there are a number of very rare tumors which may be extirpated. The polypi, however, are clinically the most important. Histologically they are mostly fibromata or fibroangiomata which produce grave symptoms, even endangering the life, and their removal, as a rule, is foilowed by a complete cure. The question as to the best method of removing them has been discussed for a long time in the past, and even now there is a wide divergence of opinion on this point among surgeons.

The difficulties of the technic lie in the obstacles encountered in obtaining access to the growths. They arise in a region which can not be attacked through any natural opening, even with instruments of special construction, without making a considerable breach in the skeleton of the face. The development of the technic of the operation shows that surgeons are more and more endeavoring to replace the operation in the dark by an anatomic preparation under the guidance of the eye. Experience shows that pieces of the growth left behind after such operations of polypi cause not only recurrences, but degenerate and even become malignant (Koenig) and, therefore, the surgeon endeavors to remove the growth radically. On the other hand, others maintain positively that such relinquished pieces disappear spontaneously, that even the whole tumor of considerable size disappears after a certain age, say twenty.five years (LafontGosselin), but it can not be discovered, a priori, what is going to be their course. Besides, there is another cause which was pre-eminently noticeable in one of my cases, calling for the most radical removal of these tumors. These fibromata are often multiple, and parts are liable to be overlooked, and these remaining small growths may develop to enormous size, as owing to the removal of part of the growth, 
they obtain room to spread. Therefore, the axiom must be: Under the guidance of the eye remove everything anatomically by preparation. If we review the history of the technic we can see that it yields more and more to this postulate: First of all, the tumor must be exposed.

When I speak of tumors of this kind. I mean only those which, on account of their considerable size, cause obstructions of the nasopharyngeal space with dyspnea, dysphagia, etc. By no means do I refer to those small growths which produce only local symptoms, as obstruction of choanes, catarrh, etc. Such large fibromata with their protuberances toward the pharynx, larynx, antrum and orbit, cause destruction of the bone, dislocation of the eyeballs, may perforate the base of the skull, and give rise to meningitis. One of the most pronounced cases I ever saw is the specimen in the Musée Dupuytren, in Paris, the picture of which has found its way into most textbooks.

To lay bare such tumors one must produce a considerable breach in the skeleton of the face. The objection to such an operation is found on the one hand in the disfigurement of the shape of the face, and on the other hand, the greatest objection is in the hemorrhage during the operation, and its consequences thereafter, by causing diseases of the lung (pneumonia), gangrene, etc. No wonder that formerly even prominent surgeons refrained from operative radical treatment, and that many still resort to palliative rather than radical methods, considering the operation too dangerous. If we start from this standpoint, we will consider that method best which in the highest degree fulfills the following requirements: 1 , total exposure of tumor; 2 , prevention of hemorrhage; 3 , least possible disfigurement and best function of the parts operated upon after the surgical interference; 4, prevention of secondary diseases.

It is no easy matter to satisfy all these requirements, but we can approach very near to the ideal. The methods used are:

1. The ligature or treatment with the écraseur, the galvanocaustic scissors or loop. This least answers the requirements, as the écraseur very often breaks, and the hemorrhage becomes excessive, and that part of the tumor remaining behind the ligature sloughs off and septic inflammation takes place, the sloughed and septic parts are aspirated into the lungs, and septic pneumonia and gangrene follow. Such methods are being abandoned more and more by modern surgeons, and in the future will not be used, we hope. The same objections hold good against all mortifying methods.

2. Electrolysis, a method which gives remarkable results in the hands of skillful operators like Nélaton, Bruns, Koenig, Karsberg, and others, is not reliable in every case. It has, however, one advantage, that it can reduce the size of large growths, and removes the mechanic obstruction symptoms, but it may be safely said that in large-sized tumors it will not remove the entire growth. That requires an enormous amount of patience on the part of the surgeon and the person treated, and when the tumor diminishes in size, it will become less and less accessible to instruments, and for this reason we may say that it is impossible to remove the tumor radically. Moreover, tumors on the antrum and orbit can not be reached at all, and they may afterward grow and become malignant. Besides, the technic accomplishment of such a treatment is very arduous, and can not be applied except in hospital oases, and perhaps only in such cases where hospital operations allow tedious experiments of this kind. In this country a patient would never consent to having forty or fifty treatments, painful and uncertain in their ultimate results, if he knew that a radical operation would remove the growth surely and rapidly. Electrolysis certainly may be of great use where we have to deal with small tumors with irritative symptoms.

3. As to the operative treatment, in reviewing the many methods advised by different surgeons, we can say that each and every one has its advantages for special cases. For large growths, however, only a few can be of use. All operations aim to get access to the polypi and, naturally, those which get the best access with the least destruction are to be preferred. All breach and flap operations have one great danger, the hemorrhage, causing anemia, which becomes dangerous as such, or by aspiration into the lung. The latter danger is more to be feared. The hemorrhage may arise from the severed vessels of the face or the vessels of the tumor, and may be prevented by ligature of the main vessels furnishing the blood, like the carotid, or by diverting the blood outward. To prevent aspiration of the blood, we can let it run out from the hanging head, or we can perform a preliminary tracheotomy with tamponade of the upper airpassages. If wo compare these two safety measures with each other, the ligature of the carotid artery seems to me preferable. It is not dangerous, but it may not be sufficient to prevent all hemorrhage. From my experience in a number of cases operated on for such purposes, I may say that it has always been sufficient. One can object, and say that if the hemorrhage comes from the tumor it comes from both sides, since the tumor receives its nourishment from both sides. In reality, however, one side is affected more than the other, and one can diagnose the affected side beforehand from the clinical symptoms, e. g., from the protruding of one eyeba!l, the destruction of the bone on one side, etc. To operate without such ligature of the main vessels, and to rely on the ligature of the single vessels as they are reached, means to operate in a pool of blood, and such operations are the most dangerous, tedious, and also the least clean. The preliminary ligature has therefore, many advan. tages. The tracheotomy is a very useful method, and Razumovski has sbown in operating on several cases that it prevented the aspiration of blood, but it is. unnecessary and under certain conditions may cause complications. Generally, we do not perform preliminary tracheotomy, because we succeed with ligature of the carotid and operation on the hanging head.

As to the formation of the breach necessary for exposure of the growth, it can be performed two ways: 1. From the mouth. Gussenbauer's method seems to me preferable to any other, because it gives the best access to the growth, and does not disfigure the face, but one still operates under difficulties, and an anatomic preparation is hardly possible. 2. From the face. The method of Ollier used by Razumovski, that is, the flapping open of the nose, does not produce large scars, but it gives insufficient access, and also the scar formation is not trifling. The nose often looks very flat, and as a notch or dent is produced in some cases, the result is very unsightly. To me the old Ferguson's or Liston's method seems the best. It fulfills all requirements. Of course I always ligate the caro- 
tid artery beforehand, so that I operate with very little loss of blood. The operation consists in osteoplastic resection of the upper jaw. An incision is made, beginning at the middle ear, crossing the face in a line underneath the eye, passing alongside the nose around the wing of the nose to the middle of the upper lip, splitting this upper lip and the soft parts of the palate right through, chiseling the bone on the malar, nasofrontal and middle palatal lines, prying the bone at its junction, and thus the whole tumor is made accessible. It may now be removed with its processes and anatomically prepared, all the hemorrhage stopped, the different parts replaced and sutured-only the two incisors being wired - otherwise only the soft parts are united, but in the most exact manner. Particular attention must be paid to this adaptation, inasmuch

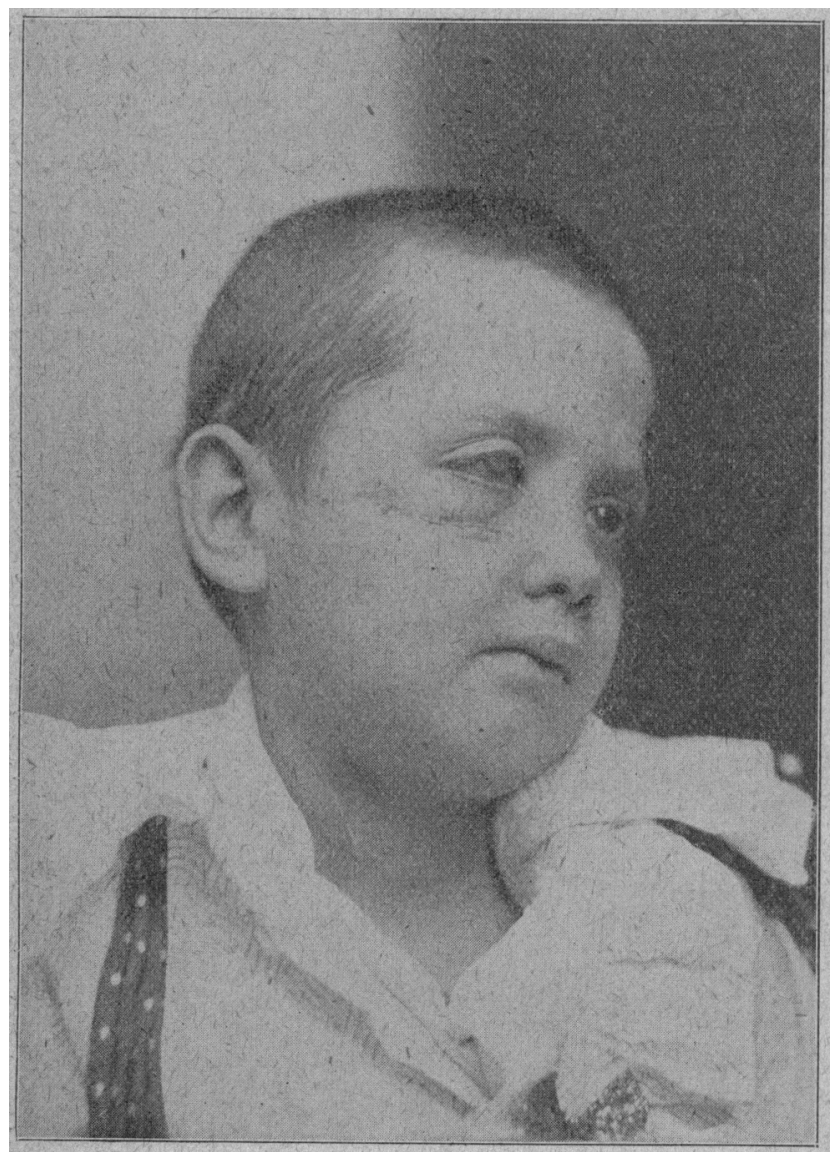

Figtre 1.

as primary union insures a good function of the parts replaced, and causes the least disfigurement. As the accompanying figure of one of the cases shows, this disfigurement is very trifling, and as the scars run all along the natural folds, they are hardly noticeable (Fig. 1).

In the same manner as these nasopharyngeal polypi, I have removed sarcomata and carcinomata of the retropharyngeal region, leaving the upper jaw, where it was not affected by disease. I must not neglect to mention one feature of this operation which caused me considerable trouble during the after-treatment. In the osteoplastic, as well as the permanent extirpation of the upper jaw, a considerable chemosis of the conjunctiva of the same side set in, and in many cases ulceration of the cornea developed. At first I could offer no explanation for this, as neither vasomotor nor any other nervous disturbance could be detected. In one of the last cases it appeared to me that the ulceration was due to pressure from the dressing. By loosening the coaptation of the lower lid and cheek, the lower lid loses its tension and droops, thus opening the slit of the eye, and leaving the cornea exposed to the friction from the dressing, which, however fine it may be, might cause ulceration of the cornea. I would, therefore, suture the eyelids temporarily, or paste them with collodium to prevent such a mishap.

Without going into details of all cases operated upon by this method, I will give briefly the history of one of the most interesting:

Case 1.-C. P., 10 years old, was brought to me by his mother, in a very critic condition, unable to walk in consequence of exhaustion. He had been sick for a long time, and as he sat in the chair, with wide open mouth, gasping for breath, the expression of his face showed hunger for air. This was due to some obstruction in the respiratory passage. The face was disfigured by protrusion of the right eyeball, and the entire right side of the face. The nose on that side did not show the usual nasolabial fold, but the bridge passed directly into the cheek. The right nostril was very much enlarged, and showed a bluish-white tumor mass protruding. The inspection of the mouth showed that the posterior portion of the pharynx was filled out by a tumor mass, depressing the soft palate and tongue and leaving only a very small space for the entrance of air and, naturally, also for food. The mother gave the following history: The child had experienced difficulty in breathing since she could remember, but it had become very annoying in the past two years. She had consulted several physicians, amonget them specialists, who advised operations and had tried to operate on the child. Dr. B., under whose care this child had been at the Clinic for Nose and Throat of the College of Physicians and Surgeons, gave me the following report: He had tried to remove the tumor through the nostril with an ecraseur, but broke the same in application. The bleeding from the tumor was so profuse that the child was almost exsanguinated, and for two days in danger of death. He consequently gave up the operation. A well-known surgeon also advised against operation on account of the dangers attending the same, so the mother decided to let the matter rest.

The expression and appearance of the boy's face reminded me so very much of the classic specimen in Dupuytren Musée, Paris, of a retromaxillary fibroma, that the diagnosis was almost intantaneous. In my judgment, the tumor was about the size of a man's fist, and must have destroyed considerable of the bone and other tissues in order to have found space for growth. I told the mother that the operation would be dangerous, but was the only effective thing to do, and she consented, even taking the chance of having the child's face disfigured.

The boy was taken to the Post-Graduate Hospital, and the operation was there performed before the class. The first step taken was a ligation of the carotid artery, in a classic manner, preparatory to the operation. Then the child was brought into Rose's position (i. e., with head hanging over the table), and an incision made, beginning at the tragus of the ear underneath the eye toward the inner angle corresponding to the nasal fold, to the middle of the upper lip around the nose; this upper lip was split right through, also the gingiva in the center, and then the palate slit clear down to one side of the uvula. A chisel was then used to divide the malar bone, the upper maxillary at its junction to the frontal in a backward direction, and the maxillary bone through the palatal process chiseled through. This last time the chisel was used to pry open the maxillary bone, and with a few movements to and fro it was raised from its place, and thereby bent to the side like a lid. The operation thus far caused some bleeding, but not one ligation of an artery of any size was necessary. The bleeding was easily controlled by the application 
of a few forceps. Now the tumor was entirely exposed, and it could be seen that it had filled out, not only the pharyngeal cavity, but also the antrum Highmori, and had with one lobule partly destroyed the inferior orbital plate, and with another partly destroyed the anterior wall of the maxillary. The tumor seemed to have its origin at the sphenoidal sinus, at least there was its closest attachment. With curved scissors it was separated all along its attachment. This separation again caused some, but not excessive, hemorrhage. Though the tumor seemed to belong to both sides of the base of the cranium, the right side seemed to furnish more circulation to the same, the hemorrhage being very light on account of the liga. ture of the carotid artery.

One peculiarity was noticed in the growth. Two entirely independent lobules of the same nature were found in the external portion of the antrum, making three tumors in all. In an operation not having such a wide opening, these two tumors would certainly have escaped notice, and would not have been removed. As it is known that such remaining tumors occasion. ally grow rapidly after operations, and even become malignant, an omission of this kind might have prevented the results of the operation. After the removal of the growth, the bleeding cavity was packed with gauze, the ends of the strips brought out through the undivided nostril, then the open skin flap of the upper jaw closely attached to its former place, and all the divided surfaces exactly sutured.

The shock of the operation was considerable, as was expected, but under careful observation the pulse remained in good condition, and the child rallied. There was no complication, and seven days afterward the child could be exhibited at a meeting of the Chicago Medical Society. One remarkable feature developed in this case, also in two others, which remained unexplained to me as well as to ophthalmologists whom I consulted. The child developed a central ulcer of the cornea, of the right eye, which resulted in an opacity necessitating iridectomy, performed by Dr. Gradle. The result of this operation, cosmetic, as well as curative, was perfect. Today, about two years after the operation, the child is perfectly well, has no perceptible disfigurement of the face, and the bones are as solidly united as before.

Cases of sarcomata and carcinomata of the retromaxillary region, naturally, have many more difficul. ties. In the tirst place, they are not so easily enucleated as the fibromata, for they must be traced in the tissues they attack, and if any chance of total and radical removal is present, it is only possible by tracing them with the guiding ey $\theta$. The malignant growths destroy the adjoining tissues not only by pressure, but by infiltration, and we have, therefore, much more destruction in these cases. We have to be satisfied with impaired functions after recovery. In one of my cases of sarcoma of the retromaxillary region, the history of which I will mention briefly below, this destruction reached such dimensions a priori, not diagnosticated, that the functional result after the operation was very poor.

Case 2.-V. K., 32 years old, a blackemith, had been suffer ing for several months. At first his physician called his com plaint neuralgia of the face. Later on he detected growth of the uvula and behind the same. He thought it was an abscess, punctured it, but the free hemorrhage showed it was a solid growth. The patient was brought to the Presbyterian Hospital, and on account of difficulty in breathing, tracheotomy was to be performed, but proved unnecessary. An incision was made into the retromaxillary tumor, some fluid withdrawn from the abscess. like growth, whereupon the man could breathe easily. In the hospital an interbuccal operation was tried by the attending surgeon, particles of the growth were removed, but the bulk of it being inaccessible was left behind, and thereupon the growth increased in size and was accompanied by the most excruciating pains.

I saw him after he had been suffering about six months, proposed a radical operation, brought him to the Post-Graduate School, and operated upon him shortly afterward. Osteoplastic resection, in the manner described above, was made, and a tumor of sarcomatous nature, about the size of a child's fist, removed. The lateral wall of the pharynx had been involved, and this was sutured, and healed by primary union, as well as all the incisions. The patient was demonstrated about six weeks afterward before the Chicago Medical Society. At that time there had been no recurrence, but in about six months intracranial symptoms showed that the tumor had not remained local, and the patient died from metastatic growth in his brain a few weeks after. This case again proves the truth of my initial remarks as to the prognosis of malignant growth.

In another class of growths of the retromaxillary region it may be more expedient to operate by temporary resection of the lower jaw. This refers to that class of tumors of the tonsils or parotids which reach into the retromaxillary space with the bulk of their growth. Such tumors are more laterally situated, and more easily diagnosed than those of the former class. It is a question whether this temporary resection of the lower jaw is necessary, or whether such tumors are more easily removed by the inframaxillary route, and either esophagotomy or pharyngotomy are performed. This must be decided by the surgeon in individual cases, according to the extent of the growth. When the bulk is in the upper pharyngeal region, even extending into the attic, it is necessary to sever the lower jaw. When it is more inferior, the inframaxillary route underneath the jaw will be sufficient. A special case described more in detail may illustrate this method of operation of retropharyngeal growths.

Case 3.-Miss H., a school teacher, had been well until about one and one half years previous to the time she consulted me. She had had a tooth extracted from her lower jaw. The ex braction was very difficult, and left a thickening of the lower jaw, which was not painful, and not very noticeable. Shortly afterward, however, a growth appeared adjoining this, gradually increasing upward, and became prominent on the left side in front of the ear, but more noticeable as a globular protruberance in the pharynx, pushing the soft palate upward on that side, also the tongue. At first the growth was rapid, but then remained stationary for a time.

She had consulted prominent surgeons of this city, who all agreed in the diagnosis of an osteosarcoma of the lower jaw, involving the adjoining tissues, perhaps the base of the skull, and advised against operation. It was at the time of Dr. Coley's publication on the effect of erysipelas and prodigiosus toxins on malignant growths that I made some experiments in this line in the Cook County Hospital. This case was referred to me by Dr. F. B. Earle, whom the patient had last consulted, as a hopeless case of sarcoma, to be given the benefit of these injections. I injected eight ounces of the erysipelas toxin (Coley) with the result that the tumor grew to such a size that the patient could hardly breathe. The tumor filled up the mouth cavity almost entirely on the side of its origin, and a large portion on the other side. She could neither swallow nor breathe, and begged me to undertake something to relieve her sufferings. I 
finally decided to make an effort to remove the growth, even if it were necessary to leave some of the tumor if I could not effect a radical cure, in order to give her a chance to breathe and eat so long as she remained alive. Previous to the operation, which was undertaken at the Post-Graduate Hospital, I ligated the external carotid artery, on the left side, in order to avoid hemorrhage. This I do in all these cases. The external incision was carried transversely along the lower jaw clear down to the bone, the jaw severed at about the middle of its transverse branch, pried open, and the tumor exposed. Strange to say, it was easily enucleated, and proved to be not a sarcoma of the lower or upper jaw, but a tumor of the parotid gland, which had grown with the small portion around the ascending branch of the lower jaw externally, with its largest portion internally, pushing before, behind and upward all the tissues which were in the way. When removed, it was about as large as two

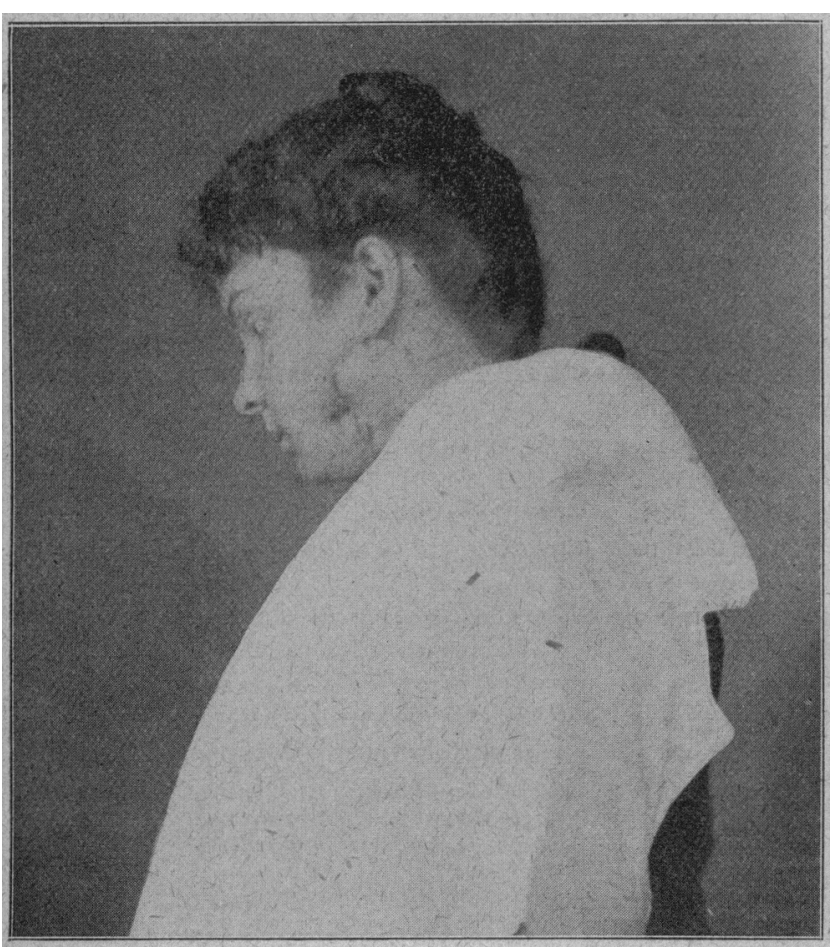

FigLRE 2.

fists. The jaw was adapted, but owing to the large cavity left by the removal of the tumor, it did not unite, and to this fact $I$ attribute the extremely tedious and very disagreeable after-treatment of the case. It had many complioations. A short time after the operation I was one night called to the hospital. The ligature of the carotid had cut through, and the interne was scarcely able to control the hemorrhage, which in a few seconds had completely covered the patient with blood. We finally succeeded in controlling it, and the very anemic patient slowly recovered from the operation. The jaw did not unite, being infected, small portions became necrotic, the two fragments placed themselves in an angular position. so the face seemed drawn to one side. The portion of the normal parotid gland had remained, and the ductus Stenonianus having been severed during the operation, large amounts of saliva poured through the cheek. I tried to bring about union of the jaw by means of dental appliances, but for a long time without success.
Finally, Dr. Goslee of the Chicago College of Dentistry succeeded in bringing about union, with a very ingenious splint. Prior to this, I operated on the patient for her salivary fistula, dissected the ductus Stenonianus with its outer opening clear down to the rest of the gland, and implanted the opening internally into the angle of the jaw, keeping it there by sutures. In this way I closed the fistula. At the present time, a little over two years after the operation, the patient is well and has returned to her former avocation. The microscopic examination of the growth had shown a mycochondro-sarcoma. Though many operations were done, the disfigurement is not very marked (Fig. 2).

If we review the subject briefly, we must acknowledge that the open method of treating such deepseated pathologic conditions as retromaxillary tumors is gaining more and more adherents among surgeons. I call attention to an article published by Prof. Jordan, in the Muenchener Medizinischer Wochenschrift, bringing forward a new method, which is still more extensive than the ordinary osteoplastic dissection. He even goes so far as to temporarily remove the external nose in order to gain access to the retromaxillary growth, and combines temporary resection of the upper jaw with temporary resection of the nose by Ollier's method. His two cases seem perfect successes, both cosmetically and therapeutically. In one case, he flapped the jaw to one side, the nose to another; in the other case, he formed one flap of nose and jaw to one side. For simple nasal polypi, even of large size, such double operations appear to me unnecessary, as the removal of the jaw in the manner previously described gives perfect success. In cases of malignant growth, however, I should advise laying open as much as possible, even to the extent of flapping both upper jaws to either side and upward, so as to lay bare the whole base of the skull, so far as can be reached by the mouth.

Whenever malignant growth in the retromaxillary region have reached such dimensions that the jaw can not be replaced, and the growth must be removed, we resort to permanent resection of the jaw. This operation, also undertaken with the preliminary ligation of the carotid artery, is very disfiguring, functionally, and cosmetically destructive. From among several cases of this kind, I shall describe one which I consider remarkable, not only on account of the extent of the operation, but also owing to the amount of plastic reconstruction.

Case 4.-Mr. R., about 40 years of age, was referred to $\mathrm{my}$ service in the surgical department of the Cook County Hospital. He had removed a tooth on the right side, as it had caused him great pain, but the swelling around the cavity did not disappear. On the contrary it grew after the extraction, and many foudroyant hemorrhages weakened the man. At the same time, difficulties in swallowing existed. On examina. tion, I found a large exulcerated growth of the right side of his jaw, extending over the tonsil, the pharynx, the right side of the tongue-not involving the tongue. The tongue could be eaeily protruded. The growth, though very extensive, did not involve any of the vital structures, and I therefore decided to remove it radically.

The operation was performed in the same manner as described in the first case, but in addition the jaw was totally removed, also the structures, so far as they were affected. The hemorrhage was very light, compared to the hemorrhage in cases where the ligation of the carotid had not been performed. The accompanying photograph shows the patient a few days after the operation (Fig. 3). 
After he had rallied from the operation, the question arose as to the method of covering the jaw defect. The whole cheek, external and internal, a portion of the pharynx, the whole upper jaw and a portion of the lower jaw were missing. The first thing I did was to unite the wound alongside the lower jaw with the remaining border of the mucous mernbrane of the

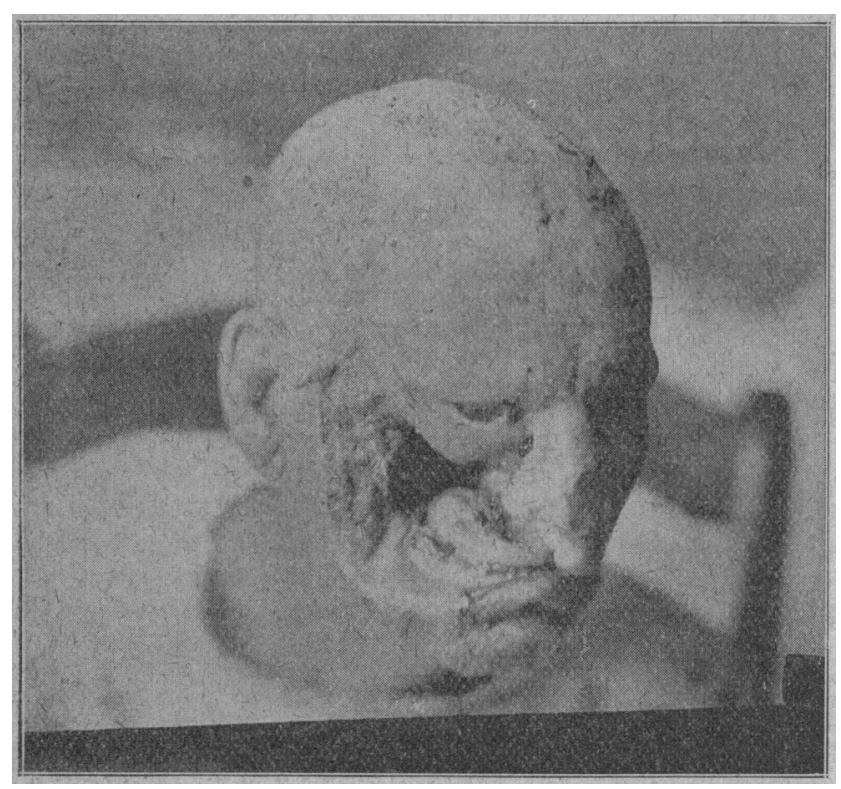

FIG, 3.-Rawlins, 45 y. Carcinoma palat, maxill. super., dextr. pharyng. Resectio maxill. super, septi nasal, ossis ethmoid, etc. Cook Co. Hospital 94. Before plastic operation.

mouth all along the jaw, thus preparing to transform a portion of the neck for a transplantation into the interior of the mouth to form the internal or mucous covering of the cheek. A few days following I inserted this flap in such a manner as to suture the

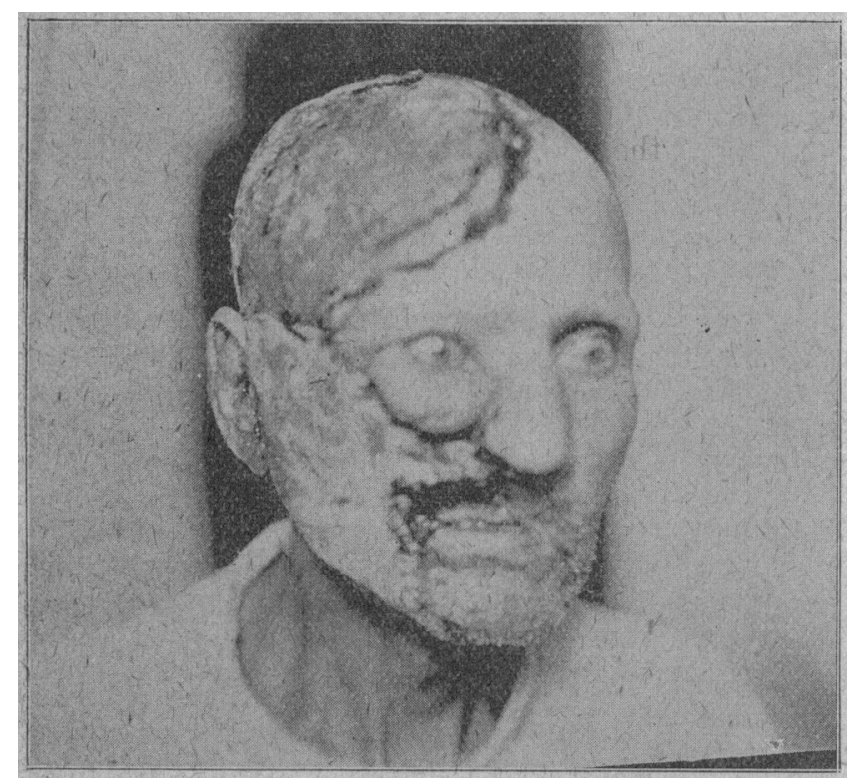

Figure 4.

flap of the neck to the wound of the pharynx and the other side of the palate, leaving a free wound outside, but covered toward the mouth opening. Two weeks later I covered this wound with the outside flap in the following manner: In order to prevent loss of blood, I made a percutaneous suture on the skull, outlining with this suture as large a flap as was necessary to form the cheek. After this suture had been put in the flap was turned down, the pedicle for the same being behind the ear. It was turned down and sutured alongside the eye, the nose around the wing to the middle of the lip, to the inner flap, which came from the neck, then to the lower lip. This was all that was necessary in order to cover the large defect. There was, however, an enormous defect on the skull, which had to be covered by Thiersch grafts. The accompanying figure (Fig. 4) shows the condition about this time. The man stood these operations very well, but his anemia was so great, and the swallowing of the discharges from the suppurating cavities of his mouth produced such destruction of his alimentary tract, that he ran down more and more, and a short time afterward died from septic fever. This case again proves the truth of my statements in the beginning of this article about malignant growths and their operations.

In conclusion, I would say with regard to the operative treatment of retromaxillary growths:

1. Retromaxillary growths should be operated with the open method, that is, osteoplastic resection.

2. To avoid hemorrhage, preliminary ligature of the carotid artery is the safest means.

3. Tracheotomy can be avoided by placing the patient in Rose's position, operating on the hanging head.

4. Non-malignant growths of small size can be treated without resections, eventually with electrolysis. Large non-melignant growths can not be treated otherwise than by resections.

5. Malignant growths of any extent give very unfavorable prognosis, and should not be treated radically except in cases of indicatio vitalis.

\section{PERSISTENT HEADACHE.}

REMOVAL, FOR RELIEF, OF AN OUNCE RIFLE-BULLET, IMBEDDED IN THE BONES OF THE RIGHT TEM-

PORAL FOSSA, WHERE IT HAD LAIN

UNSUSPECTED FOR THIRTYFOUR YEARS.

BY SWAN M. BURNETT, M.D., Ph.D.

Professor of Ophthalmology and Otology, Georgetown University; Ophthalmologist to the Georgetown University Hospital and

to the Children's and Providence Hospitals; Director of the Eye and Ear Clinies at the Central Dispensary and Emergency Hospital. WASHINGTON, D. C.

Mr. W. G., of Howardsville, Va, at the age of 17 years enlisted, June 16,1863, in the Second Virginia Cavalry. In the sixteen engagements in which he took part, from that time until July 3 , he escaped unhurt; but at the battle of Cashtown, on that date, he was twice wounded. The first wound was in the right shoulder, which, while disabling him as to the use of his gun, did not render him unconscious or drive him from the field; and while cheering his comrades on in the fight, he received his second wound, in the head, which prostrated him and rendered him unconscious for some minutes. He was carried to the rear, and remained as prisoner in the hands of the Federal troops. He was sent to a hospital in Providence, R. I., where his shoulder wound assumed a very serious aspect, and he was in a critical condition for several weeks. As soon as he was able to observe things after regainining consciousness on the field, he noticed that the sight of his 